\title{
High Frequency Performance of Piezoelectric Diaphragms for Impedance-Based SHM Applications ${ }^{\dagger}$
}

\author{
Guilherme Rezende and Fabricio Baptista * \\ Laboratory of Transducers and Data Acquisition, Department of Electrical Engineering, School of \\ Engineering, São Paulo State University (UNESP), Bauru 17033-360, Brazil \\ * Correspondence: f.baptista@unesp.br; Tel.: +55 14 3103-6764 \\ + Presented at the 7th Electronic Conference on Sensors and Applications, 15-30 November 2020; Available \\ online: https://ecsa-7.sciforum.net/.
}

Published: 15 November 2020

\begin{abstract}
Piezoelectric transducers are used in a wide variety of applications, including damage detection in structural health monitoring (SHM) applications. Among the various methods for detecting structural damage, the electromechanical impedance (EMI) method is one of the most investigated in recent years. In this method, the transducer is typically excited with low frequency signals up to $500 \mathrm{kHz}$. However, recent studies have indicated the use of higher frequencies, usually above $1 \mathrm{MHz}$, for the detection of some types of damage and the monitoring of some structure's characteristics that are not possible at low frequencies. Therefore, this study investigates the performance of low-cost piezoelectric diaphragms excited with high frequency signals for SHM applications based on the EMI method. Piezoelectric diaphragms have recently been reported in the literature as alternative transducers for the EMI method and, therefore, investigating the performance of these transducers at high frequencies is a relevant subject. Experimental tests were carried out with piezoelectric diaphragms attached to two aluminum bars, obtaining the impedance signatures from diaphragms excited with low and high frequency signals. The analysis was performed using the real part of the impedance signatures and two basic damage indices, one based on the Euclidean norm and the other on the correlation coefficient. The experimental results indicate that piezoelectric diaphragms are feasible for the detection of structural damage at high frequencies, although the sensitivity decreases.
\end{abstract}

Keywords: piezoelectric diaphragms; SHM; electromechanical impedance; high frequency; performance; sensitivity

\section{Introduction}

Throughout the world there is a large number of civil and mechanical structures, such as urban infrastructures and large means of transport, in constant use by the population that can suffer damage due to wear and environmental influences. Therefore, the detection of damage at an early stage on these structures is of global interest, as it permits the reduction of maintenance costs and provides security to users.

For this purpose, structural health monitoring (SHM) systems have been investigated [1,2]. Such systems should monitor the structure, preferably in real time or periodically, and detect structural damage before its performance is impaired and the safety of users is at risk. Although SHM systems can be used on a wide variety of structures, studies reported in the literature have mainly focused on aeronautical structures [3] and civil infrastructures [4].

In order for the structure not to be impaired during monitoring, damage detection must be performed using a non-destructive testing (NDT) technique which is minimally invasive to the 
monitored structure. There are many NDT techniques reported in the literature that can be applied to SHM systems, such as Lamb waves [5] and acoustic emission [6]. In this work, the structural damage detection is based on the electromechanical impedance (EMI) technique, which uses piezoelectric transducers attached to the monitored structure operating simultaneously as both sensor and actuator [7].

This technique is usually performed at low frequencies, below $500 \mathrm{kHz}$. Recent studies indicate, however, that piezoelectric transducers should be excited at high frequencies, generally higher than $1 \mathrm{MHz}$, for the detection of some types of damage or to monitor some characteristics of the structure [8]. Thereby, an analysis of piezoelectric transducers at high frequencies is necessary. Therefore, the performance of piezoelectric transducers excited with high frequency signals for structural damage detection based on the electromechanical impedance is analyzed in this work.

\section{Electromechanical Impedance Technique}

As mentioned previously, damage detection based on the EMI technique consists of attaching a piezoelectric transducer to a structure to be monitored. The transducer's impedance signatures as a function of frequency are obtained for the structure at a healthy state and for a state in which the structure is possibly damaged.

There are several models which have been proposed to relate the transducer's electrical impedance and the structure's mechanical impedance. For thin transducers and small structures, as is this work's case, a one-dimensional model is satisfactory, so that the electrical impedance of the piezoelectric transducer attached to the structure is given by [9]:

$$
Z_{E}(f)=\frac{1}{j 2 \pi f C}\left(1-\frac{d_{31}^{2}}{s_{11}^{E} \varepsilon_{33}^{T}} \frac{Z_{S}(f)}{Z_{S}(f)+Z_{P}(f)}\right)^{-1}
$$

where $Z_{E}(f)$ is the transducer's electrical impedance, $Z_{S}(f)$ is the monitored structure's mechanical impedance, $Z_{P}(f)$ is the transducer's mechanical impedance, $f$ is the frequency, $C$ is the transducer's capacitance, $j$ is the imaginary unit, $d_{31}^{2}$ is the piezoelectric constant, $s_{11}^{E}$ is the elastic compliance and $\varepsilon_{33}^{T}$ is the dielectric constant. The superscripts $E$ and $T$ indicate constant electric field and constant stress, respectively, and the subscripts 1 and 3 represent the axes of the natural coordinate system of the piezoelectric material under the one-dimensional assumption.

According to Equation (1), any variation to the structure's mechanical impedance caused by structural damage will result in a corresponding variation in the transducer's electrical impedance. Therefore, the structure's integrity can be monitored by measuring and analyzing the transducer's electrical impedance.

Typically, damage detection is performed through damage indices, comparing two impedance signatures. One is obtained for a healthy state of the structure and is used as reference (baseline), while the other signature is obtained during the monitoring of the structure. One of the damage indices used in this work is the root mean square deviation (RMSD), based on the Euclidean norm, which is given by [2]:

$$
R M S D=\sum_{f=f_{I}}^{f_{F}} \sqrt{\frac{\left[Z_{2}(f)-Z_{1}(f)\right]^{2}}{Z_{1}(f)^{2}}}
$$

where $Z_{1}(f)$ is the impedance signature obtained for a healthy state of the structure and $Z_{2}(f)$ is the impedance signature obtained during the monitoring of the structure, $f_{I}$ is the initial frequency, and $f_{F}$ is the final frequency.

The other damage index used in this work is the correlation coefficient deviation metric $(C C D M)$, based on the correlation coefficient, which is given by [10]:

$$
C C D M=1-\left|\frac{\sum_{f=f_{I}}^{f_{F}}\left[Z_{1}(f)-\bar{Z}_{1}\right]\left[Z_{2}(f)-\bar{Z}_{2}\right]}{\sqrt{\sum_{f=f_{I}}^{f_{F}}\left[Z_{1}(f)-\bar{Z}_{1}\right]^{2}} \sqrt{\sum_{f=f_{I}}^{f_{F}}\left[Z_{2}(f)-\bar{Z}_{2}\right]^{2}}}\right|,
$$

where $\bar{Z}_{1}$ and $\bar{Z}_{2}$ are the impedance signatures' mean values obtained for a healthy state of the 
structured and during the monitoring of the structure, respectively. In this work, the real part of the impedance signatures was used.

In the next section, the experimental setup used to verify the transducer's performance for damage detection at high frequencies is presented.

\section{Experimental Setup}

To verify the piezoelectric transducer's sensitivity to damage detection at high frequencies, two aluminum plates were used as structures to be monitored, one with dimensions of $500 \mathrm{~mm} \times 119 \mathrm{~mm}$ $\times 1 \mathrm{~mm}$ (Structure A) and the other with dimensions of $304 \mathrm{~mm} \times 196 \mathrm{~mm} \times 1 \mathrm{~mm}$ (Structure B), supported by rubber blocks. In this study, low-cost piezoelectric diaphragms, which are mass-produced sound components by several manufacturers, were evaluated. Three types of piezoelectric diaphragms were used as transducers, all from Murata Manufacturing: a 7BB-15-6, with an active piezoelectric element with a diameter of $10 \mathrm{~mm}$; a 7BB-20-6, with an active piezoelectric element with a diameter of $14 \mathrm{~mm}$; and a 7BB-35-3, with an active piezoelectric element with a diameter of $25 \mathrm{~mm}$. One transducer of each type was attached to each structure using cyanoacrylate adhesive, so that all diaphragms were at the center of the plate and $50 \mathrm{~mm}$ from one of the plate's ends. Structural damage was simulated by means of attaching two steel nuts to the structures, one with dimensions of $9 \mathrm{~mm} \times 4 \mathrm{~mm}$ (Large Damage) and the other with dimensions of 4 $\mathrm{mm} \times 1.5 \mathrm{~mm}$ (Small Damage). The nuts were used one at a time, coupled to the structure at its center point. The experimental setup is shown in Figure 1.

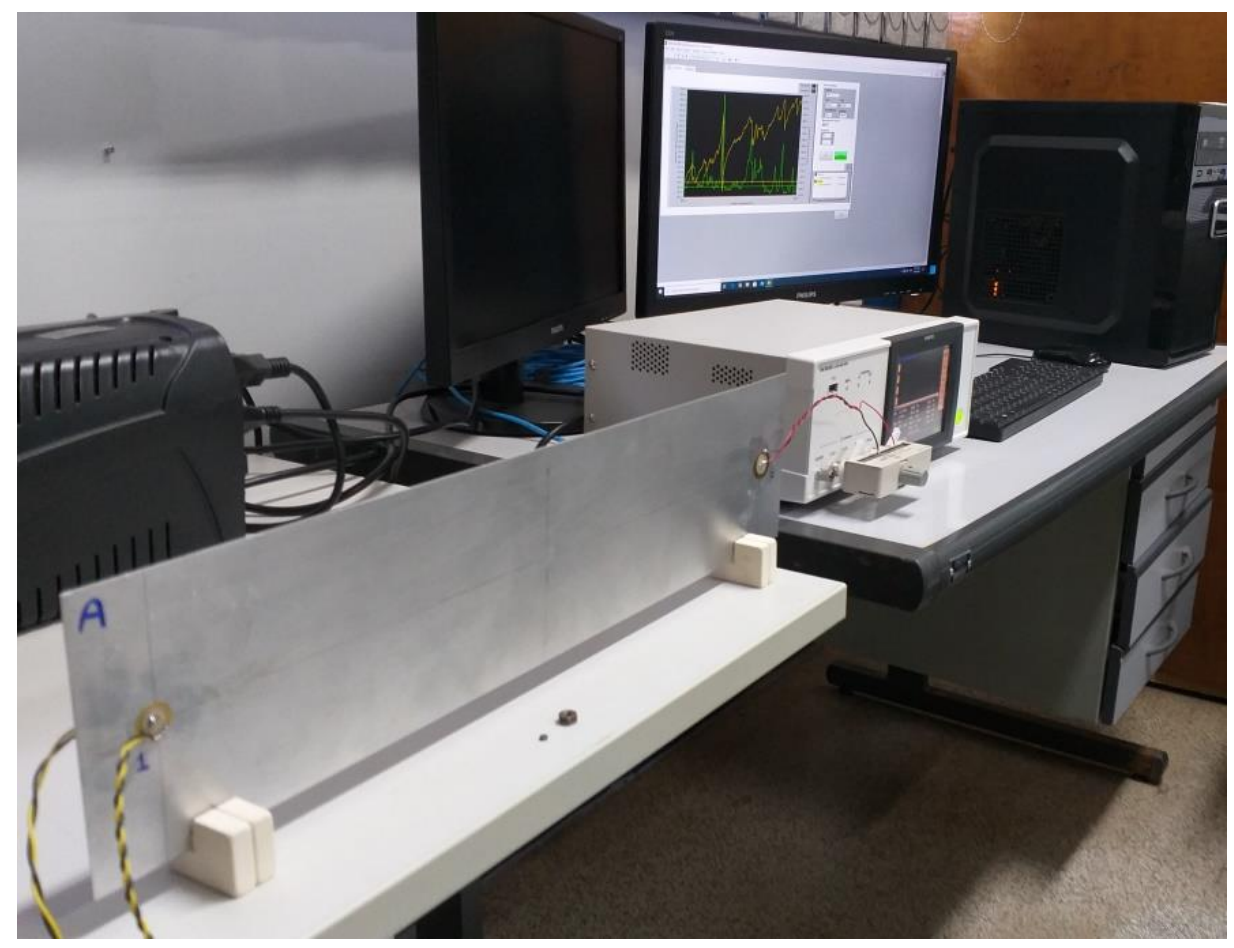

Figure 1. Experimental setup.

To measure the transducers' electrical impedance, an IM3536 LCR meter from HIOKI connected to a personal computer was used, with excitation voltage of $0.06 \mathrm{~V}$ rms. A low voltage was chosen so that the high frequency current was within the limits of the LCR meter. The transducers' impedance signatures were obtained for low frequencies, in a range from $60 \mathrm{kHz}$ to $80 \mathrm{kHz}$, and for high frequencies, in a range from $1 \mathrm{MHz}$ to $1.2 \mathrm{MHz}$, in order to compare their performances at both situations. With the impedance signatures, the RMSD and CCDM indices were calculated in sub-bands of $1 \mathrm{kHz}$, for low frequencies, and in sub-bands of $10 \mathrm{kHz}$, for high frequencies. The obtained results are presented and discussed in the next section. 


\section{Results and Discussion}

As mentioned previously, structural damage changes the transducer's electric impedance, causing the electrical impedance signature to change in relation to the baseline. This can be seen in Figure 2, which shows the real part of the impedance signatures obtained for the largest transducer on Structure B, for both low and high frequencies and for small and large damages.

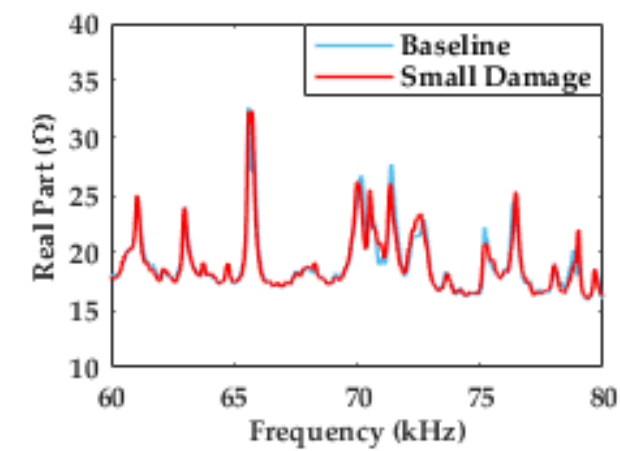

(a)

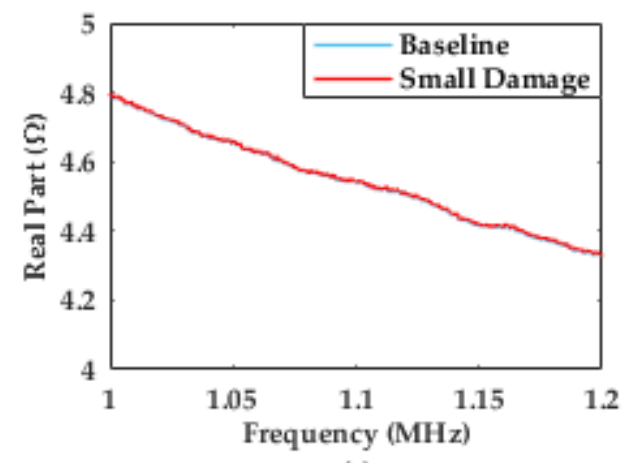

(c)

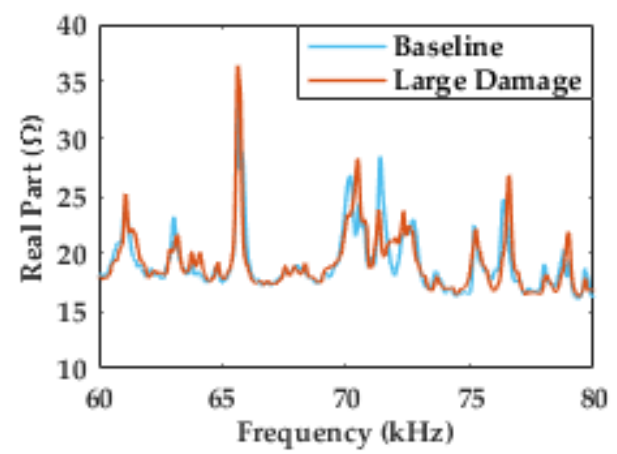

(b)

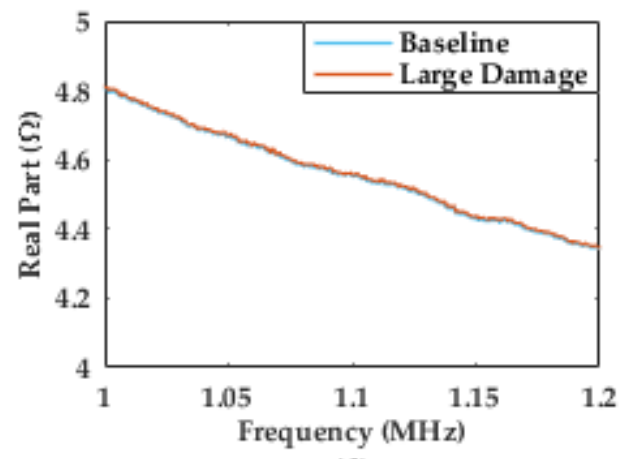

(d)

Figure 2. Impedance signatures of the largest transducer on structure B for (a) small damage and (b) large damage for low frequency, and for (c) small damage and (d) large damage for high frequency.

Figure 3 shows the calculated RMSD indices for the same situation described above.

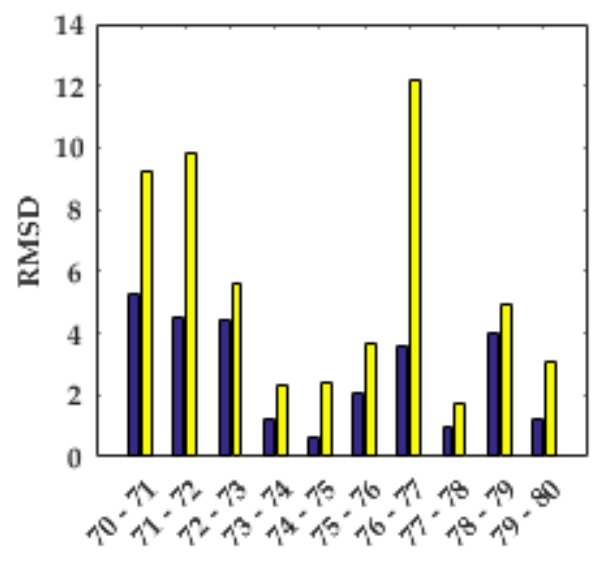

Frequency $(\mathbf{k H z})$

(a)

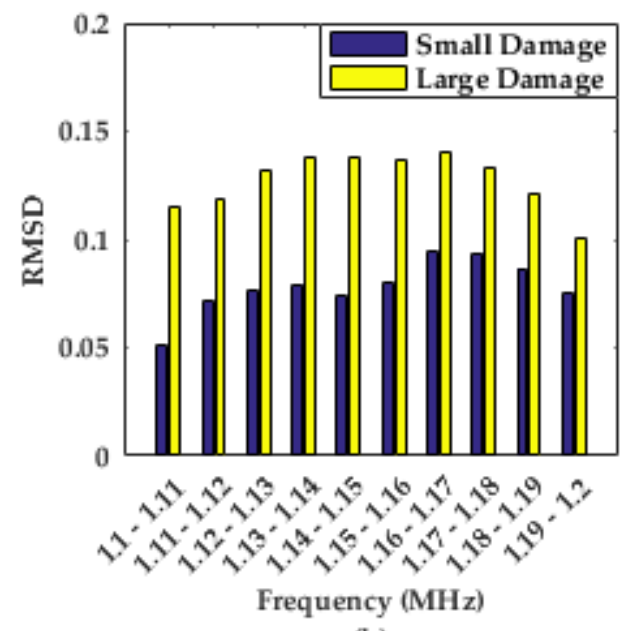

(b)

Figure 3. RMSD indices obtained from the largest transducer on structure B for small and large damages for (a) low frequency and (b) high frequency.

Figure 4 shows the calculated CCDM indices for the same situation described above. 


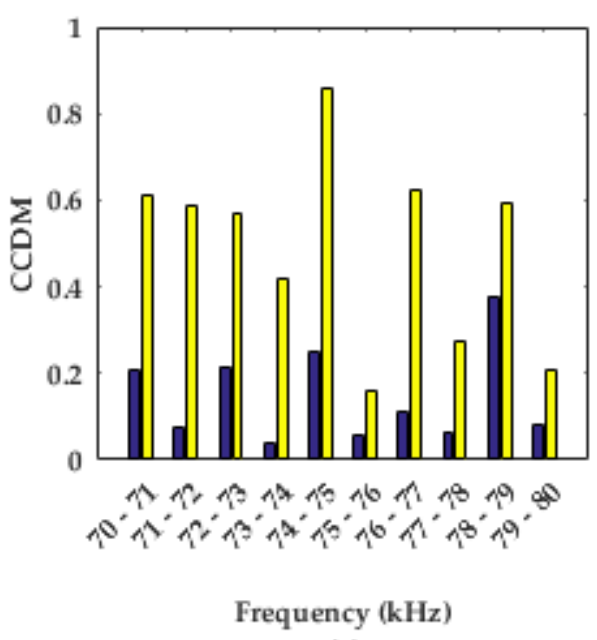

(a)

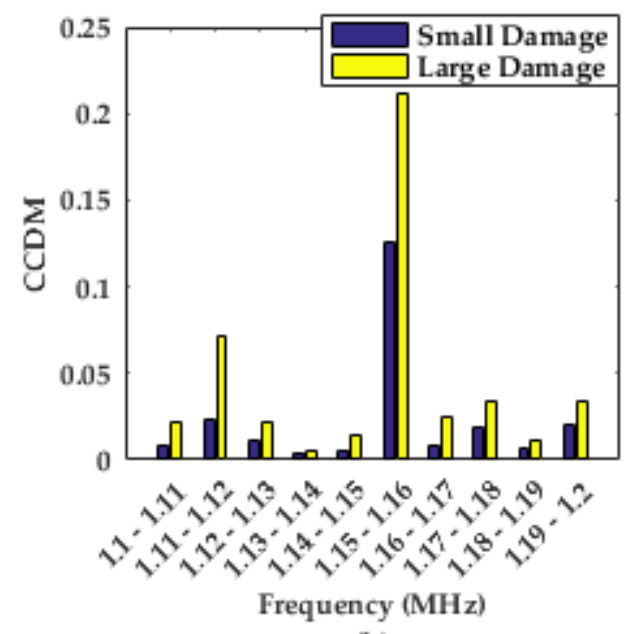

(b)

Figure 4. CCDM indices obtained from the largest transducer on structure B for small and large damages for (a) low frequency and (b) high frequency.

The RMSD and CCDM indices are presented in the range from $70 \mathrm{kHz}$ to $80 \mathrm{kHz}$ for low frequency and in the range from $1.1 \mathrm{MHz}$ to $1.2 \mathrm{MHz}$ in order to facilitate the visualization and comparation of the results.

As can be seen in Figure 2, there is almost no qualitative difference between the baseline and the impedance signature for the damaged structure at high frequency, for both damages. However, variations in the impedance signatures can be quantitatively observed by calculating damage indices.

In Figures 3 and 4, we can see that the RMSD and CCDM indices calculated at low frequencies were considerably higher than those calculated at high frequencies, for both damages. This result pattern was observed for all transducers on both structures. Therefore, it is seen that piezoelectric diaphragms are capable of detecting and quantifying damage at high frequencies, but the sensitivity is significantly lower.

\section{Conclusions}

This work presents a comparative analysis between the piezoelectric diaphragms' sensitivity for structural damage detection at low and high frequencies in SHM systems based on the electromechanical impedance technique. Two aluminum plates were used as structures to be monitored, each with three transducers of different sizes. Two sizes of damage were simulated on the structures and the transducers' electrical impedance signatures were obtained for low and high frequencies.

The damage indices obtained at high frequencies were lower than those obtained at low frequencies. However, despite the low values, these indices vary according to the size of the structural damage. Therefore, the experimental results indicate that piezoelectric diagrams are capable of detecting and quantifying structural damage at high frequencies, although the sensitivity is lower compared to low frequencies.

Acknowledgments: The authors are grateful to FAPESP-São Paulo Research Foundation (grant \#2019/11358-0) and the Brazilian National Council for Scientific and Technological Development-CNPq (grant 400824/2016-7, and grant 312069/2018-9).

Conflicts of Interest: The authors declare no conflict of interest. 


\section{References}

1. Annamdas, V.; Bhalla, S.; Soh, C. Applications of structural health monitoring technology in Asia. Struct. Health Monit. 2017, 16, 324-346.

2. Farrar, C.R.; Worden, K. Structural Health Monitoring: A Machine Learning Perspective; Wiley: Chichester, UK, 2013; p.654.

3. Swindell, P.; Doyle, J.; Roach, D. Integration of structural health monitoring solutions onto commercial aircraft via the Federal Aviation Administration Structural Heath Monitoring Research Program. In Proceedings of the 43rd Annual Review of Progress in Quantitative Nondestructive Evaluation, Atlanta, GA, USA, 17-22 July 2017; AIP Conference Proceedings, 2017.

4. Li, H.; Ren, L.; Jia, Z.; Yi, T.; Li, D. State-of-the-art in structural health monitoring of large and complex civil infrastructures. J. Civ. Struct. Health Monit. 2016, 6, 3-16.

5. Shan, S.; Cheng, L.; Li, P. Adhesive nonlinearity in Lamb-wave-based structural health monitoring systems. Smart Mater. Struct. 2017, 26, 25019.

6. Pearson, M.; Eaton, M.; Featherston, C.; Pullin, R.; Holford, K. Improved acoustic emission source location during fatigue and impact events in metallic and composite structures. Struct. Health Monit. 2017, 16, 382399.

7. Albakri, M.I.; Tarazaga, P.A. Electromechanical impedance-based damage characterization using spectral element method. J. Intell. Mater. Syst. Struct. 2017, 28, 63-77.

8. Kamas, T.; Tekkalmaz, M. Coupled field modeling of E/M impedance of piezoelectric wafer active sensor for cataphoretic coating thickness measurement. Smart Mater. Struct. 2017, 26, 1-13.

9. Liang, C.; Sun, F.P.; Rogers, C.A. Coupled electro-mechanical analysis of adaptive material systems-determination of the actuator power consumption and system energy transfer. J. Intell. Mater. Syst. Struct. 1994, 5, 12-20.

10. Marqui, C.R.; Bueno, D.D.; Baptista, F.G.; Vieira Filho, J.; Santos, R.B.; Lopes Junior, V. External disturbance effect in damage detection using electrical impedance. In Proceedings of the International Modal Analysis Conference, Orlando, FL, USA, 4-7 February 2008; Curran: Red Hook, NY, USA, 2008.

Publisher's Note: MDPI stays neutral with regard to jurisdictional claims in published maps and institutional affiliations.

(C) 2020 by the authors. Submitted for possible open access publication under the terms and conditions of the Creative Commons Attribution (CC BY) license (http://creativecommons.org/licenses/by/4.0/). 